\title{
Expression levels of matrix metalloproteinases in ascites of patients with ovarian cancer and the relationship with pathological characteristics of ovarian cancer
}

\author{
Shuna S, Ping L, Junfei $W^{*}$ \\ Department of Obstetrics and Gynecology, Baogang Hospital, Baotou, Inner Mongolia, China
}

Received: March 30, 2019

DOI: $10.5430 /$ dcc.v6n2p12
Accepted: May 21, 2019

Online Published: June 10, 2019

\begin{abstract}
Objective: To explore the expression levels of matrix metalloproteinase-2 (MMP-2) and matrix metalloproteinase-9 (MMP-9) in ascites in ovarian tumor and provide a theoretical basis for the diagnosis and prognosis evaluation of ovarian cancer ascites. Methods: ELISA was used to detect the levels of MMP-2 and MMP-9 in ascites samples from 73 cases of patients with malignant ovarian tumor, and RIA was used to detect the expression level of CA125 in the serum in these patients.

Results: The expression levels of MMP-2 and MMP-9 in ascites in malignant ovarian tumor were higher than those in ascites in benign ovarian tumor $(t=8.08,10.39, p<.01)$, and the difference was of statistical significance. The expression levels of MMP-2 and MMP-9 in patients with stage III and IV malignant ovarian tumors were higher than those in patients with stage I and II malignant ovarian tumors, and the difference was statistically significant $(t=2.75,2.75, p<.05)$. There was no statistically significant difference in the expression levels of MMP-2 and MMP-9 among the patients with different pathological types, different histological grades, lymph node metastasis or not, different ascites volumes and different residual lesions $(p>.05)$. The sensitivities of detecting MMP-2 and MMP-9 in ascites were 76.0\% and 88.0\%, respectively, which were significantly higher than those of ascites cytological examinations $\left(\chi^{2}=4.61,12.74, p<.05\right)$, but in comparison with serum CA125, there was no statistically significant difference $(p>.05)$. The specificities of detecting MMP-2 and MMP-9 in ascites were 78.3\% and 82.6\%, respectively, which were significantly lower than those of ascites cytological examinations $\left(\chi^{2}=5.61,4.38, p<.05\right)$, but in comparison with serum CA125, there was no statistically significant difference $\left(\chi^{2}=1.64,2.68, p<.05\right)$.

Conclusions: The levels of MMP- 2 and MMP-9 in ascites may be markers for the differential diagnosis of benign and malignant ovarian lesions, and they are related to the prognosis in patients with malignant ovarian tumors.
\end{abstract}

Key Words: Malignant ovarian tumor, Matrix metalloproteinase, Ascites

\section{INTRODUCTION}

The malignant ovarian tumor is one of common tumors occurring in female genital organs. ${ }^{[1]}$ Patients with this type of tumor often develop ascites, which may clinically result from many causes. The golden standard for the differential diagnosis of benign or malignant tumor is ascites cytological examination. However, because of the low sensitivity showed by ascites cytological examination, patients with malignant

\footnotetext{
*Correspondence: Junfei W; Email: ffyu666727@qq.com; Address: Department of Obstetrics and Gynecology, Baogang Hospital, Baotou, Inner Mongolia 014010, China.
} 
ovarian tumors who only develop ascites and show negative results in this examination have to only receive symptomatic treatments and repeated cytological examinations instead of timely surgical operations or chemotherapies. It is imperative to find a specific and sensitive tumor marker to improve the prognosis and the quality of life in patients with ovarian cancer. $^{[2]}$ This research was designed to explore the role of MMP-2 and MMP-9 in the diagnosis and prognosis evaluation of ascites in malignant ovarian tumor by detecting their levels in ovarian cancer patients with ascites and analyzing their correlation to clinicopathological characteristics.

\section{MAterials AND Methods}

\subsection{General information}

73 cases of hospitalized patients in Baogang hospital from December of 2015 to December of 2017 were selected as research objects. There were 50 cases of malignant ovarian tumors, 7 cases of endometriosis cyst of the ovary, 16 cases of ovarian benign tumors (including 7 cases of mucinous cystadenoma and 9 cases of serous cystadenoma). All cases were confirmed by pathological examinations after operation. In the group of malignant ovarian tumors, according to different standards, these cases can be divided as follows: clinical staging: 10 cases of stage I and stage II and 40 cases of stage III and IV; ascites volume: 20 cases whose ascites volume was no more than $1,000 \mathrm{ml}$ and 30 cases whose ascites volume was more than 1,000 ml; pathological type: 10 cases of peritoneal carcinoma, 25 cases of serous cystadenoma, 8 cases of mucinous cystadenoma, 3 cases of clear cell carcinoma, 2 cases of poor differentiated adenocarcinoma and 2 cases of endometrial carcinoma; histological grading: 32 cases of poor differentiated tumors and 18 cases of moderate and high differentiated tumors; lymph node metastasis or not: excluding 4 cases which were not recorded during operation, there were 10 cases of patients with lymph node metastasis and 30 cases of patients with no lymph node metastasis; residual lesion: there were 31 cases of patients with the residual lesion whose size was less than $2 \mathrm{~cm}$ and 19 cases of patients with the residual lesion whose size was no less than $2 \mathrm{~cm}$. There were 8 out of 50 cases of patients with malignant ovarian tumors who were not given ascites cytological examinations. All samples were acquired by means of laparoscopic surgery or laparotomy operation, and centrifuged at the rotate speed of 3,000 r/min for $10 \mathrm{~min}$, with the supernatant taken and placed in the refrigerator at $-70 \circ \mathrm{C}$ for the experimental use.

\subsection{Experimental methods}

The detection of MMP-2 and MMP-9 in ascites: ELISA was applied to the detection, and the microplate reader was used to measure OD value (wave length $450 \mathrm{~nm}$ ). The detection

Published by Sciedu Press of serum CA125: Radio immunoassay was applied to the detection, with $35 \mathrm{KU} / \mathrm{L}$ considered as the positive standard.

\subsection{Statistical analysis}

SPSS 17.0 software was applied to statistical treatment, and the comparison in the means between two samples was made by use of $\bar{X} \pm \mathrm{s}$, with $t$-test applied. One-way ANOVA was applied to the comparison in the means among multiple samples. $p<.05$ was considered as a standard to judge whether the difference was of statistical significance.

\section{Results}

\subsection{The comparison of MMP-2 and MMP-9 levels in as-} cites in 3 types of ovarian tumors

There was a statistically significant difference in the comparison of MMP-2 and MMP-9 levels in ascites in 3 types of ovarian tumors. The further comparison showed that MMP-2 and MMP-9 levels in ascites in malignant ovarian tumor had a statistically significant difference from those in ascites in endometriosis cyst of the ovary and benign ovarian tumor ( $p$ $<.05$ ), while there was no statistically significant difference in MMP-2 and MMP-9 levels in ascites between endometriosis cyst of the ovary and benign ovarian tumor $(p>.05)$ (see Table 1).

Table 1. The comparison in MMP-2 and MMP-9 levels in ascites in 3 types of ovarian tumors $(\bar{X} \pm \mathrm{s})$

\begin{tabular}{llll}
\hline Tumor Type & $\mathbf{n}$ & MMP-2 (ng/ml) & MMP-9 (ng/ml) \\
\hline Malignant ovarian tumor & 50 & $348.43 \pm 76.01$ & $11.14 \pm 3.89$ \\
Endometriosis cyst of the ovary & 7 & $222.04 \pm 71.47$ & $4.92 \pm 1.92$ \\
Benign ovarian tumor & 16 & $190.36 \pm 62.50$ & $4.06 \pm 1.60$ \\
$F$ Value & & 33.08 & 32.12 \\
$p$ Value & & $<.0001$ & $<.0001$ \\
\hline
\end{tabular}

3.2 The effect of clinicopathological characteristics of malignant ovarian tumor on MMP-2 and MMP-9 levels in ascites

3.2.1 The comparison in MMP-2 and MMP-9 levels in ascites among all pathological types of malignant ovarian tumors

According to statistical testing, there was no statistically significant difference in MMP-2 and MMP-9 levels in ascites among patients with different pathological types of malignant ovarian tumors, which indicated that MMP-2 and MMP-9 levels in ascites in patients with malignant ovarian tumor were not related with the pathological type of tumors (see Table 2).

\subsubsection{The relationship of MMP-2 and MMP-9 with other clinicopathological characteristics}

MMP-2 and MMP-9 levels in stage III and IV malignant ovarian tumors were higher than those in stage I and II, and 
the difference was of statistical significance $(p<.05)$. As to other clinicopathological characteristics, there was no statistically significant difference (all $p>.05$ ) (see Table 3 ).

Table 2. The comparison in MMP-2 and MMP-9 levels among different pathological types of malignant ovarian tumors $(\bar{X} \pm \mathrm{s})$

\begin{tabular}{llll}
\hline Pathological type & $\mathbf{n}$ & MMP-2 (ng/ml) & MMP-9 (ng/ml) \\
\hline Serous cystadenoma & 25 & $336.83 \pm 71.78$ & $11.31 \pm 3.73$ \\
Mucinous cystadenoma & 8 & $365.26 \pm 82.71$ & $11.09 \pm 4.68$ \\
Peritoneal carcinoma & 10 & $365.86 \pm 73.82$ & $12.05 \pm 3.77$ \\
Others & 7 & $345.77 \pm 94.71$ & $11.14 \pm 3.89$ \\
$F$ Value & & 0.49 & 0.12 \\
$p$ Value & $>.05$ & $>.05$ \\
\hline
\end{tabular}

3.3 The comparison in the authenticity and consistency among MMP-2, MMP-9, ascites cytological examination and serum CA125

The data in Table 4 showed that, among 4 indicators, MMP-9 had a highest Kappa value, which was close to 0.70; the next was MMP-2; but ascites cytological examination and serum CA125 both had a low Kappa value $(<0.4)$. It was indicated that the application of MMP-9 to the differential diagnosis of benign or malignant tumor had a best authenticity and consistency, which was superior to the application of the other 3 indicators. The difference was of statistical significance $\left(\chi^{2}\right.$ $=10.79, p<.05)$.

Table 3. The relationship of MMP-2 and MMP-9 with other clinicopathological characteristics $(\bar{X} \pm \mathrm{s})$

\begin{tabular}{|c|c|c|c|c|c|c|c|}
\hline Type & $\mathbf{n}$ & MMP-2 (ng/ml) & $t$ Value & $p$ Value & MMP-9 (ng/ml) & $t$ Value & $p$ Value \\
\hline \multicolumn{8}{|c|}{ Clinical staging } \\
\hline I, II & 10 & $292.90 \pm 69.02$ & 2.75 & $<.01$ & $8.30 \pm 2.59$ & 2.75 & $<.01$ \\
\hline III, IV & 40 & $362.32 \pm 71.92$ & & & $11.85 \pm 3.86$ & & \\
\hline \multicolumn{8}{|c|}{ Histological grade } \\
\hline Low & 32 & $363.50 \pm 70.86$ & 1.92 & $>.05$ & $11.07 \pm 3.96$ & 0.15 & $>.05$ \\
\hline High & 18 & $321.65 \pm 79.44$ & & & $11.25 \pm 3.88$ & & \\
\hline \multicolumn{8}{|c|}{ Lymph node metastasis } \\
\hline Existed & 10 & $360.91 \pm 88.06$ & 0.35 & $>.05$ & $12.12 \pm 3.92$ & 0.70 & $>.05$ \\
\hline None & 36 & $351.42 \pm 72.39$ & & & $11.13 \pm 3.98$ & & \\
\hline \multicolumn{8}{|c|}{ Ascites volume } \\
\hline$>1,000 \mathrm{ml}$ & 30 & $351.31 \pm 84.08$ & 0.33 & $>.05$ & $11.01 \pm 3.99$ & 0.26 & $>.05$ \\
\hline$\leq 1,000 \mathrm{ml}$ & 20 & $344.12 \pm 63.86$ & & & $11.31 \pm 3.82$ & & \\
\hline \multicolumn{8}{|c|}{ Residual lesion } \\
\hline$>2 \mathrm{~cm}$ & 19 & $362.77 \pm 78.28$ & 1.05 & $>.05$ & $10.48 \pm 3.35$ & 0.41 & $>.05$ \\
\hline$\leq 2 \mathrm{~cm}$ & 31 & $339.65 \pm 74.51$ & & & $11.31 \pm 4.23$ & & \\
\hline
\end{tabular}

Table 4. The comparison in sensitivity and specificity of detecting MMP-2 and MMP-9 in ascites with other detecting methods (n)

\begin{tabular}{|c|c|c|c|c|c|c|c|c|}
\hline \multirow{2}{*}{ Group and indicator } & \multicolumn{2}{|c|}{ MMP-2 } & \multicolumn{2}{|c|}{ MMP-9 } & \multicolumn{2}{|c|}{ Ascites cytological examination } & \multicolumn{2}{|c|}{ Serum CA125 } \\
\hline & + & - & + & - & + & - & + & - \\
\hline Case Group & 38 & 12 & 44 & 6 & 23 & 27 & 39 & 11 \\
\hline Control Group & 5 & 18 & 4 & 19 & 0 & 23 & 9 & 14 \\
\hline Sensitivity & 0.76 & & 0.88 & & 0.55 & & 0.78 & \\
\hline Specificity & 0.78 & & 0.83 & & 1.00 & & 0.61 & \\
\hline Kappa & 0.50 & & 0.69 & & 0.35 & & 0.38 & \\
\hline
\end{tabular}

Note. Patients with malignant ovarian tumor were included in the case group, and the patients with benign ovarian lesion (including endometriosis cyst of the ovary and benign ovarian cyst) were included in the control group. 


\section{Discussion}

\subsection{Matrix metalloproteinase and ascites in malignant ovarian tumor}

The infiltration and transfer of malignant tumor results from many genes and changes, and it is a cascade reaction in which multiple genes, steps and phases interact with each other. ${ }^{[3]}$ Matrix metalloproteinase (MMP) is a type of enzyme which is highly conserved in the process of natural evolution. ${ }^{[4]}$ It is closely related with the invasion and transfer of ovarian tumor, and its effect is achieved by degrading basal lamina and extracellular matrix ${ }^{[5]}$ and then affecting tissue remodeling. ${ }^{[6]}$ Ovarian cancer is characterized by peritoneal metastasis, ${ }^{[7]}$ and the appearance of ascites indicates the occurrence of peritoneum metastasis in malignant ovarian tumor. ${ }^{[8,9]}$ A certain research shows that the levels of MMP2 and MMP-9 in the serum are obviously higher in multiple types of malignant tumors, indicating that the formation of malignant tumor is associated with MMP. ${ }^{[10]}$ Some relevant literatures show that there are three aspects on the mechanism of MMP to promote the formation of ascites in malignant tumor: 1) The extracellular matrix and basal lamina which work as histological barriers are degraded, so that tumor cells migrate to the peritoneum; 2) Some related proteins with a certain bioactivity are activated to stimulate tumor cells to migrate to the peritoneum; 3) MMP is interacted with VEGF to promote neovascularization and accelerate the tumor to grow and migrate to the peritoneum, leading to the formation of ascites. ${ }^{[11]}$ This result indicates that the detection of MMP-2 and MMP-9 levels in ascites can be a new method to diagnose ascites in benign and malignant ovarian tumor.

Currently, dozens of markers have been applied to the differential diagnosis of benign and malignant ovarian tumors, and the commonly used include CA125, HE4, VEGF and so on. ${ }^{[12]}$ The detection of a single tumor marker has a low sensitivity in the identification of benign and malignant ascites. ${ }^{[13]}$ The experimental results show that the detection of MMP-2 and MMP-9 levels in ascites can be a good indicator in the identification of benign and malignant ovarian lesions, and it can also verify the accuracy of serum CA125 detection.

\subsection{MMP and the prognosis of malignant ovarian tumor}

The prognosis of malignant ovarian tumor is related with various factors, including clinical staging, histopathological type, histological grading, ascites volume, ${ }^{[14]}$ lymph node metastasis, the size of residual lesion and so on. The experimental results show that MMP-2 and MMP-9 levels in ascites in malignant ovarian tumor are up-regulated with the clinical stage increases, which indicates that MMP-2 and MMP-9 promote peritoneal metastasis of malignant ovarian tumor and provide a favorable environment for the extensive plantation of tumor cells in the peritoneal cavity. MMP-2 levels in ascites in malignant ovarian tumor is higher in the group of ascites volume more than $1,000 \mathrm{ml}$, the poor differentiation group, the group of residual lesion with the size of $2 \mathrm{~cm}$ above, the group with lymph node metastasis than those in the group of ascites volume no more than 1,000 ml, the moderate and high differentiation group, the group of residual lesion with the size of no more than $2 \mathrm{~cm}$, the group with no lymph node metastasis, but the difference is of no statistical significance. MMP-9 level in ascites has a rising trend in malignant ovarian tumor with/without lymph node metastasis, but the difference is of no statistical significance. There is no rising trend in the group of ascites volume more than $1,000 \mathrm{ml}$, the poor differentiation group and the group of residual lesion with the size of $2 \mathrm{~cm}$ above in comparison with the group of ascites volume no more than $1,000 \mathrm{ml}$, the moderate and high differentiation group and the group of residual lesion with the size of no more than $2 \mathrm{~cm}$. It is speculated that MMP-9 level is also affected by angiogenesis, cell adhesion and other various factors except for the degradation of histological barriers and the activation of relevant proteins with a certain bioactivity (which can promote cell migration and infiltration). ${ }^{[15]}$

In brief, the in-depth research on the mechanism of the infiltration and transfer of malignant ovarian tumor will provide a new thought for the effort in the diagnosis, prognosis and targeted therapy of malignant ovarian tumor.

\section{CONFLicts OF INTEREST Disclosure}

\section{REFERENCES}

[1] Lanmei Z. Application status of laparoscopy in the diagnosis and treatment of malignant ovarian tumor. Chinese Journal for Clinicians. 2014; 42(3): 3-5.

[2] Yan Z, Qi C. Research progress on the relationship of MMP with the occurrence and development of ovarian cancer. China Journal of Cancer Prevention and Treatment. 2015; 42(12): 1257-1261.
[3] Huizi T, Feifei L, Xu H. Research of PEA3 Transcription Factor in the Invasion and Metastasis of Ovarian Cancer. Medical Recapitulate. 2014; 20(18): 3317-3319.

[4] Zhanping W, Jinhui W, Xianghong J, et al. Expression and significance of the matrix metalloprofeinase inhibitor RECK gene in the ovarian cancer cell strains. Chinese Journal of Birth Health \& Heredity. 2015; 23(3): 14-26.

[5] Bin L, Zhengdong G, Yunhui L, et al. Clinical significance of serum 
and urine MMP-9 in patients with ovarian cancer. Chinese Journal of Clinical Oncology. 2013; 40(5): 287-289.

[6] Chuan Q, Yiya S, Yimeng L, et al. Expression of RACK1 protein and its relationship with MMP-2 in ovarian cancer. Maternal and Child Health Care of China. 2013; 30(29): 5066-5068.

[7] Liu M. Analysis on CD4+CD25+ Treg/CD4+T cells contents and significance in ascites and peripheral blood of ovarian cancer patients. Maternal and Child Health Care of China. 2016; 31(22): 4871-4874.

[8] Correa RJ, Valdes YR, Peart TM, et al. Combination of AKT inhibition with autophagy blockade effectively reduces ascites-derived ovaria cancer cell viability. Carcinogenesis. 2014; 35(9): 1951-1961. PMid:24562574. https://doi.org/10.1093/carcin/bgu049

[9] Nik NN, Vang R, Shihle M, et al. Origin and pathogenesis of pelvic (ovarian, tubal, and primary peritoneal) serous carcinoma. Annu Rev Pathol. 2014; 9(1): 27-45. PMid:23937438. https: //doi.org/10.1146/annurev-pathol-020712-163949

[10] Davidson B, Stavnes HT, Hellesylt E, et al. MMP-7 is a highly specific negative marker for benign and malignant mesothelial cells in serous effusions. Hum Pathol. 2016; 47(1): 104-108. PMid:26520416. https://doi.org/10.1016/j.humpath.2015.08.020
[11] Fathy M, Al Ansary M, Zakaria M, et al. Role of vascular endothelial growth factor (VEGF) in diagnosis of pleural effusion of different origins. Egypt J Chest Dis Tubercu. 2014; 63: 611-615. https://doi.org/10.1016/j.ejcdt.2014.02.008

[12] Mingluan L, Wei C, Yefei L, et al. Expression and clinical significance of HE4 and CA125 protein in tumor tissue and serum of patients with ovarian cancer. Maternal and Child Health Care of China. 2013; 28(24): 4023-4026.

[13] Huan G, Xiangyu D, Lu Y. Differential diagnostic value of simultaneous determination of tumor markers in ascitic fluid and serum and their ratios for differentiation of benign from malignant ascites. China Journal of Modern Medicine. 2011; 21(14): 1595-1599.

[14] Chen CY, Chang HP, Ng KK, et al. Long-term disease-free survival in three ovarian cancer patients with a single relapse. Eur J Gynaecol Oncol. 2012; 33(3): 321-323.

[15] Wintzell M, Hjerpe E, Avail Lundqvist E, et al. Protein markers of cancer-associated fibroblasts and tumor-initiating cells reveal subpopulations in freshly isolated ovarian cancer ascites. BMC Cancer. 2012; 12(1): 359. PMid:22901285. https://doi.org/10.1186/ 1471-2407-12-359 